\title{
Cognitive and social aspects of adaptation to a communication partner
}

\section{Introduction}

Kristina Thiele ${ }^{1,3}$, Anouschka Foltz ${ }^{1,2}$, Marieluise Bartels ${ }^{1,2}$, Dunja Kahsnitz ${ }^{1,2}$ \& Prisca Stenneken ${ }^{1,2,3}$

${ }^{1}$ Clinical Linguistics, Bielefeld University; ${ }^{2}$ Collaborative Research Center 673 "Alignment in Communication", Bielefeld University ${ }^{3}$ Center of Excellence "Cognitive Interaction Technology" (CITEC), Bielefeld University

$>$ Communication is a socially highly relevant form of joint action

Well-studied phenomenon: Speakers verbally adapt to each other (Branigan etal., 2005

$>$ Adaptation can be found on various linguistic levels (e.g. lexical, syntactic).

$>$ Adaptation may contribute to communicative success (Pickering \& Garrod, 2004).

Studies suggest that the interlocutor's perceived language skills affect adaptation rates (vanova etal., 2007; Branigan etal., in press). But what about other factors?

Research Question:

To what extent do speakers' linguistic skills and cognitive capacities influence syntactic adaptation?

To what extent do social and situational aspects influence lexical adaptation?

\section{Confederate Scripting to Study Adaptation}
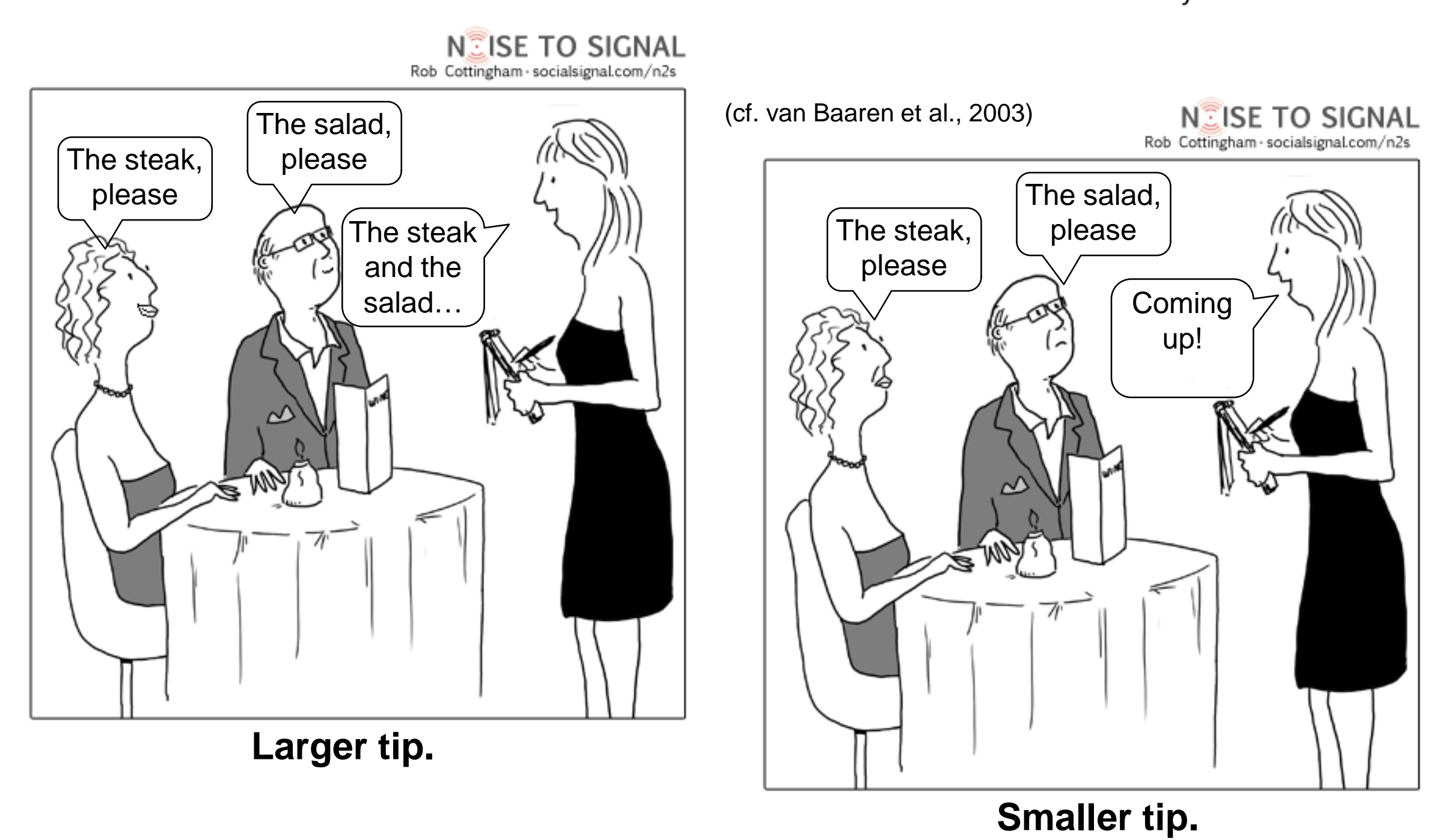

1. Confederate describes prime picture

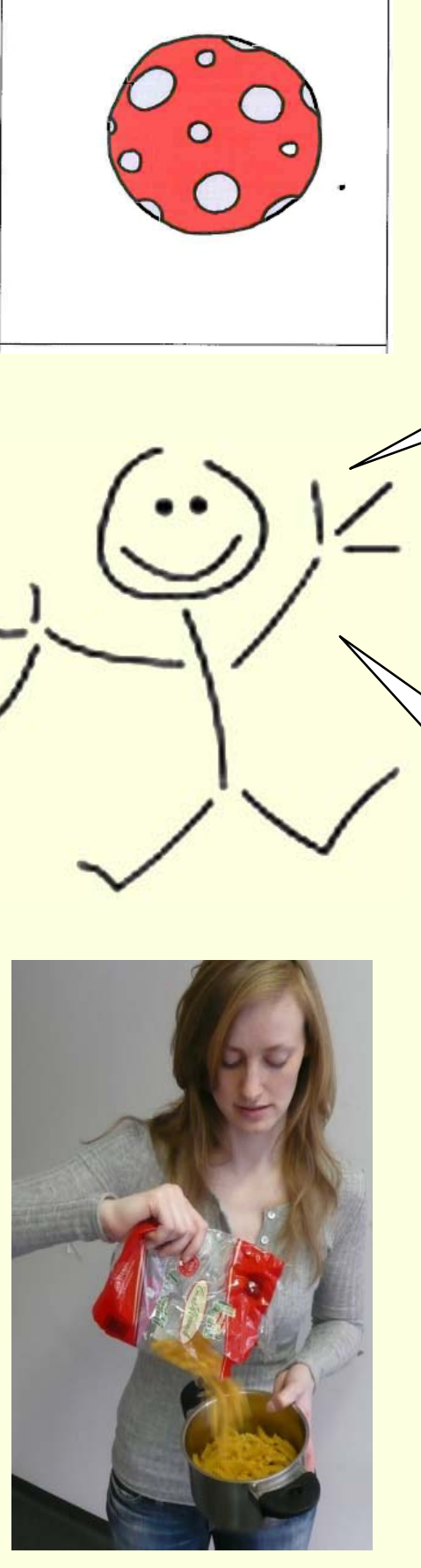

The woman
The red ball.

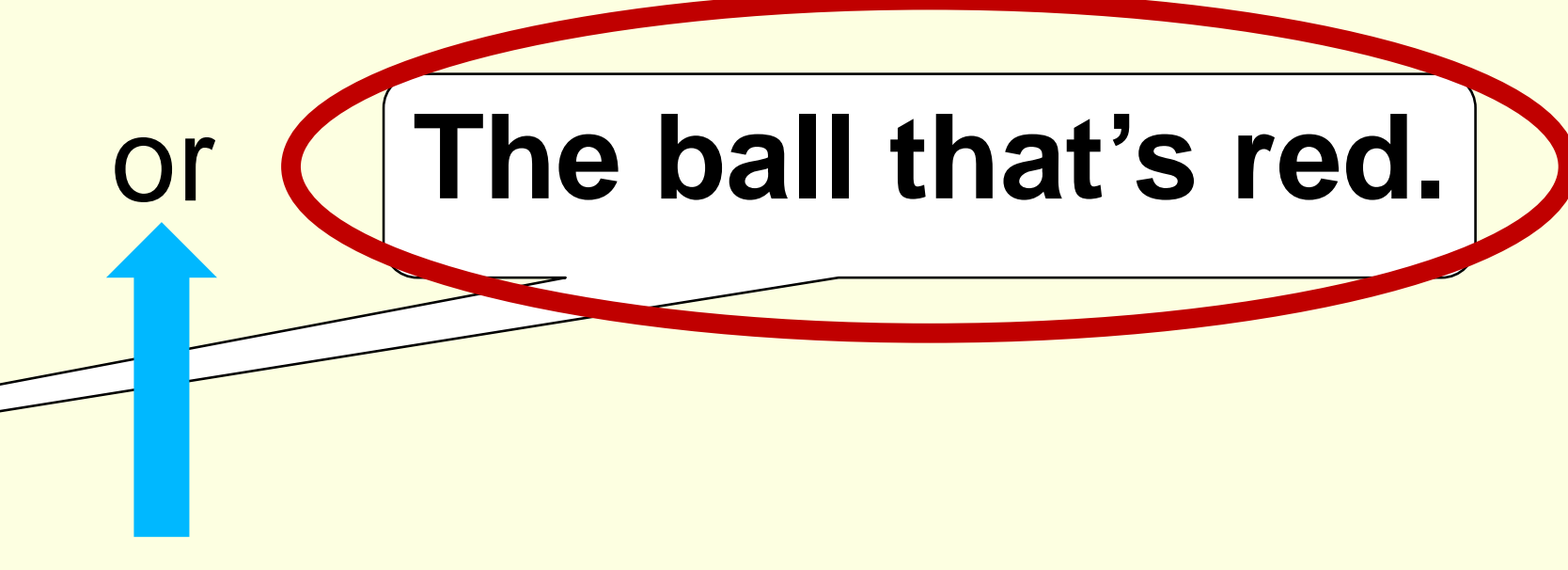

alternative SYNTACTIC structures

is cooking

pasta. dispreferred LEXICAL term in German (preferred term: noodles)
2. Participant describes target picture

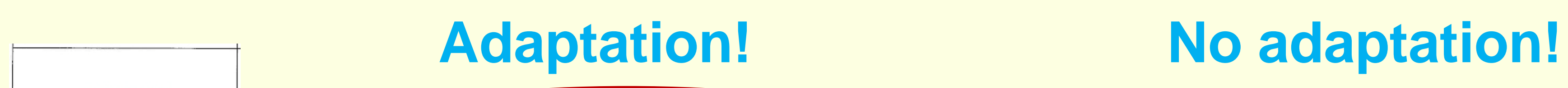

Adaptation!

No adaptation!

\section{Experiment 1}

Syntactic adaptation in children: Morpho-syntactic abilities vs. working memory capacity?

> Participants 19 (12 male, 7 female) native German-speaking children (mean age = 4;9; $S D=0 ; 5$ )

$>$ Confederate scripting with alternative syntactic structures.

Additional Tests

$>$ Taken from three standardized and norm-referenced language-development test batteries

$>$ Morpho-syntactic abilities (mean score of five tests)

Working memory (mean score of four tests)

\section{Results}

> Children adapted to both prime structures, but reliably more to "the red ball" (Figure $1, p<0.01$ ). Interesting: Adaptation to the more complex syntactic structure ("the ball that's red")!

$>$ Morpho-syntactic abilities: No effect of adaptation to complex structure (Figure 2).

Working memory: Effect of adaptation to complex structure: Higher working memory capacity =

more adaptation; lower working memory capacity = less adaptation (Figure $2, p<0.05$ )

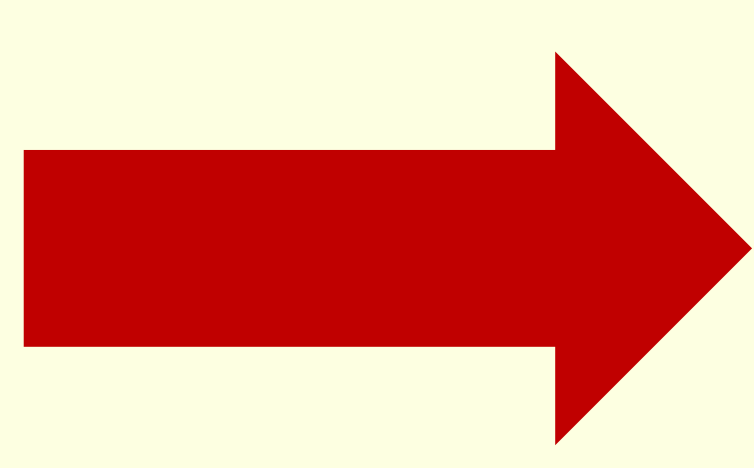

Preliminary analyses:

$>$ When taking children's age into account: Same effect of working memory capacity.

$>$ BUT also effect of morpho-syntactic abilities: Higher morpho-syntactic skills = LESS adaptation; lower morpho-syntactic skills = MORE adaptation.

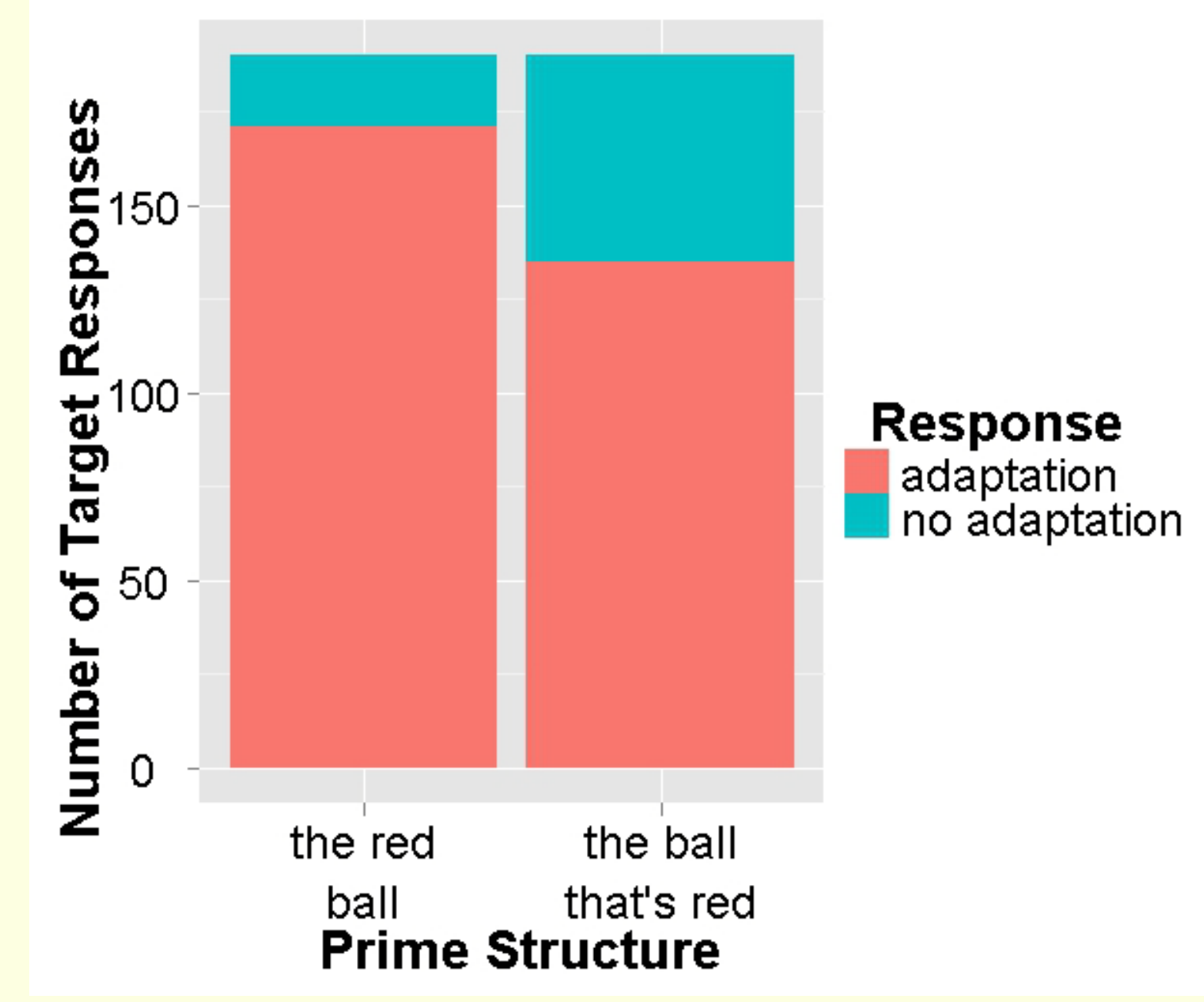

Figure 1: Adaptation in children's responses.

or The green bear.

\section{Experìment 2}

Lexical adaptation in adults: Perceived language skills vs. situational pleasantness vs. personal closeness?

> Participants 23 (12 male, 11 female) native German-speaking Bielefeld University students ( mean age $=25.30 ; S D=5.33$ )

$>$ Confederate scripting with dispreferred lexical terms and two different confederates (native vs. non-native German speaker) Additional Tests

$>$ Picture description pretest to determine lexical preference of experimental prime words

Questionnaire asking about: confederates' perceived language skills, situational pleasantness, personal closeness to confederate

\section{Results}

The use of a dispreferrred lexical term can be experimentally induced: Participants produced reliably more dispreferred terms in the experiment compared to the pretest (Figure $3, p<.001$ )

Marginal effect of confederates' identity on dispreferred term adoption rates: Numerically higher adoption rates with the non-native compared to the native confederate $(p<.1)$

Reliable positive correlation between perceived personal closeness and dispreferred-term adoption rates:

High personal closeness = more adoption; low personal closeness $=$ less adoption (Figure $4, p<.01$ )

Neither perceived language skills nor situational pleasantness showed a reliable correlation with dispreferred-term adoption rates.

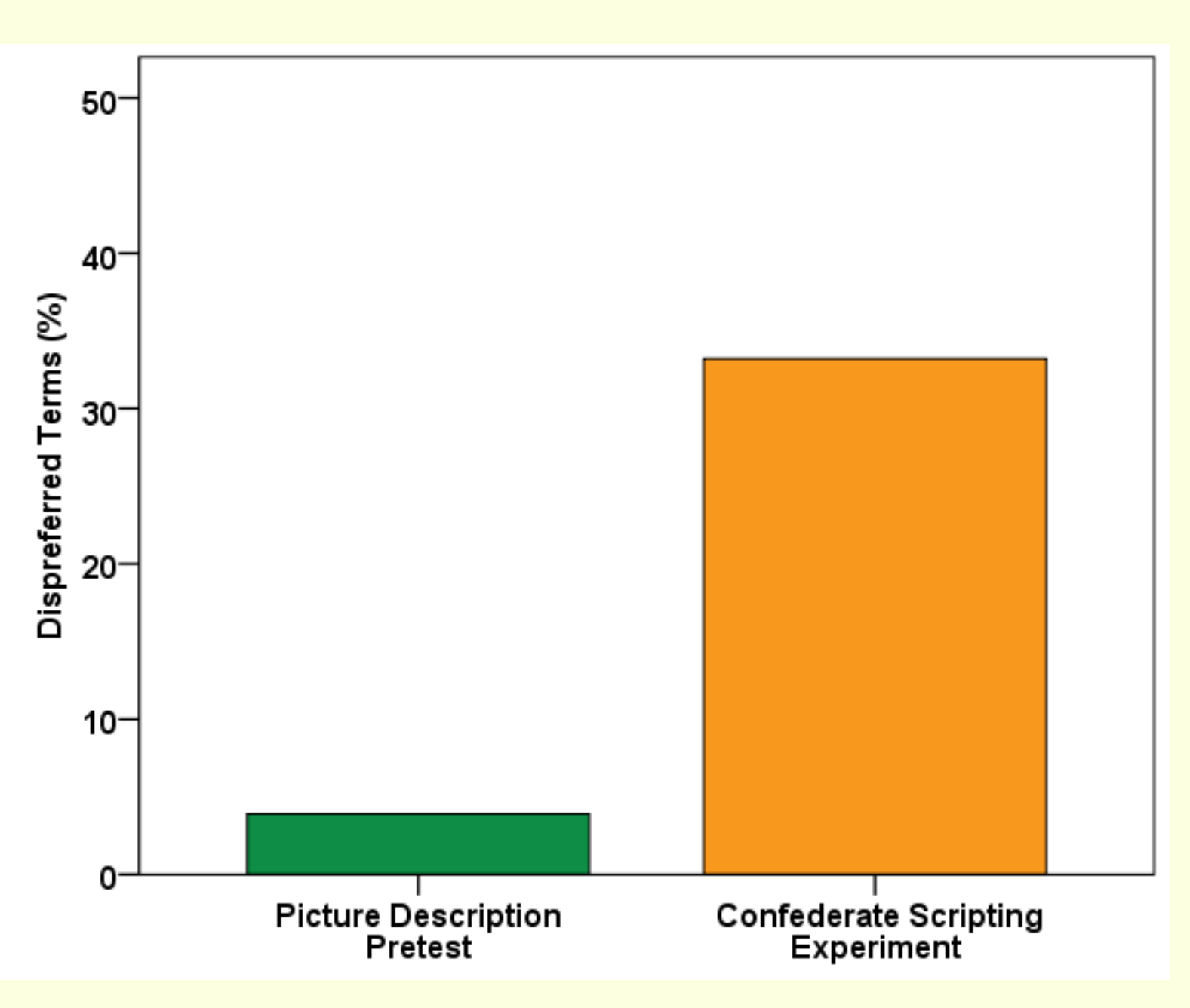

Figure 3: Dispreferred term production rates: Pretest vs. Experiment.

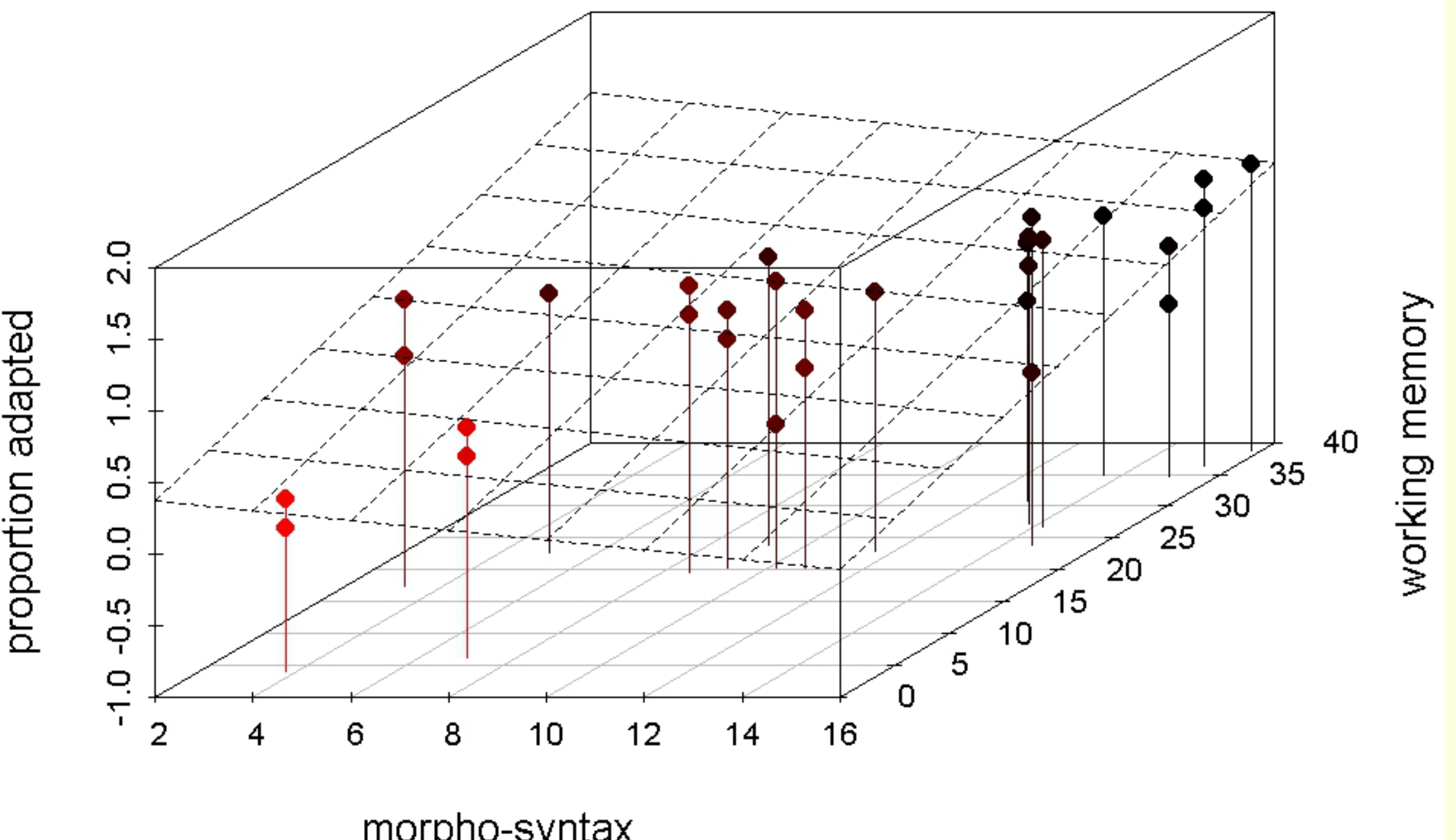

Figure 2: 3D-scatterplot showing the relationship bewr

\section{Discussion and Conclusions}

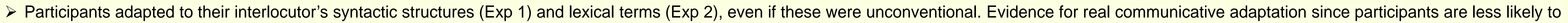
have used these unconventional terms and structures on their own.

Experiment 1:

$>$ Morpho-syntactic skills only had an effect on adaptation of syntactic structures if children's age was a factor in the statistical analysis

$>$ Working memory capacity robustly affected syntactic adaptation: Suggests that working memory capacity can limit people's ability to adapt.

Experiment 2:

$>$ Marginally more adaptation of lexical terms if the conversational partner was a non-native speaker: Evidence for strategic component of adaptation

$>$ Contrast to previous studies: No effect of perceived language skills on adaptation rates.

$>$ BUT positive correlation between perceived personal closeness and adoption rates: Evidence that personal closeness (rather than perceived language skills) affects adaptation.

Conclusions

The results of both experiments suggest that social-strategic and cognitive factors influence the amount of adaptation that may contribute to successful communication.

In addition, cognitive and situational factors may influence adaptation behavior more strongly than linguistic factors.

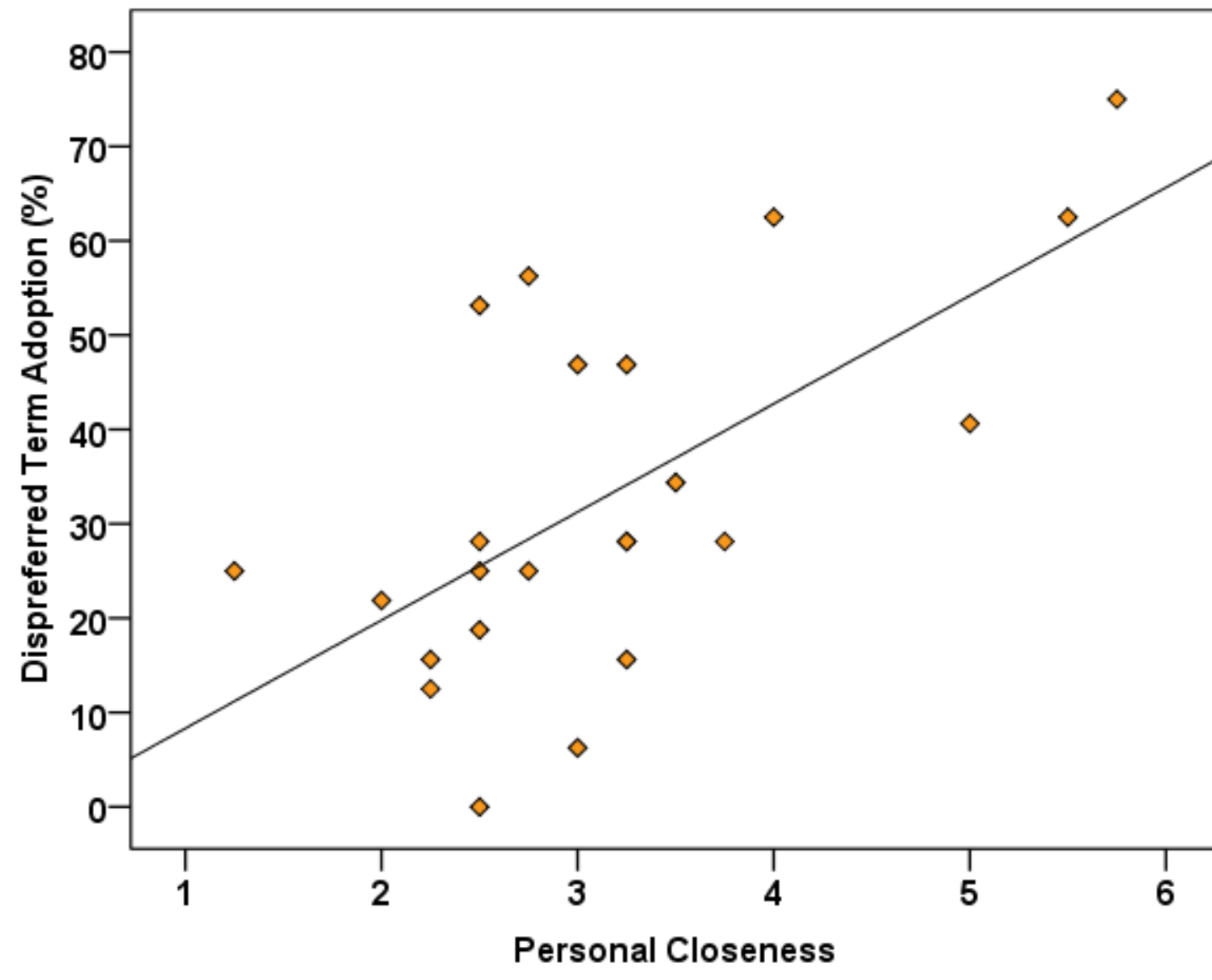

Figure 4: Scatterplot showing correlation between (a) 